\title{
Utilisation de la spectrométrie dans le proche infrarouge pour estimer la teneur en acides gras volatils du jus de rumen
}

\author{
J Aufrère, D Graviou \\ INRA, station de recherche sur la nutrition des herbivores, Theix, \\ 63122 Saint-Genès-Champanelle, France
}

Afin de développer une méthode rapide de détermination de la teneur en acide acétique (C2), propionique (C3) et butyrique (C4) du jus de rumen, nous avons comparé l'utilisation de la spectrométrie dans le proche infra-rouge (SPIR), par transmission, à la chromatographie en phase gazeuse (CPG), habituellement utilisée.

Les jus de rumen ont été prélevés avant et à différentes heures après le repas sur 4 moutons alimentés avec de la luzerne verte, puis avec les ensilages préparés à partir de ce même fourrage. Ils ont été centrifugés (27 $000 \mathrm{~g}, 2$ fois), puis conservés dans $10 \%$ d'une solution d'acide orthophosphorique à $5 \%$ avant l'acquisition du spectre dans le proche infrarouge. Les teneurs en $\mathrm{C2}$, C3, C4 des 54 échantillons, déterminées par CPG selon la méthode de Jouany (1982), variaient respectivement de 40,6 à $111,3 \mathrm{mM}$, de 8,0 à $28,1 \mathrm{mM}$ et de 3,3 à $11,3 \mathrm{mM}$. Le programme ISI a été utilisé pour établir les relations entre les spectres infrarouges et les valeurs de laboratoire (CPG), en mode 'MPLS' et à l'aide d'un modèle de régression 'stepwise', à des fins d'interprétation.
Avec le modèle MPLS, les corrélations sont établies en prenant en compte la totalité du spectre ; la calibration établie avec 54 échantillons est très précise (tableau I) comme l'avaient déjà observé Dhanoa et al (1990), mais sa validité n'a pu être contrôlée avec d'autres échantillons. L'utilisation du modèle 'stepwise' montre que la zone de longueur d'onde la plus explicative du spectre est $2258 \mathrm{~nm}$ pour le C2, et $2298 \mathrm{~nm}$ pour le $\mathrm{C} 3$, ce qui est en accord avec les pics observés pour les concentrations de constituants purs ; les bandes de combinaison $\mathrm{CH}, \mathrm{OH}$ sont retrouvées dans la zone $2000-2500 \mathrm{~nm}$ ainsi que dans la zone $1650-1700 \mathrm{~nm}$.

En conclusion, l'utilisation de la SPIR pour déterminer la concentration en acides gras volatils du jus de rumen semble très prometteuse, mais devra être confortée par l'étude d'un plus grand nombre d'échantillons.

Dhanoa MS, Barnes RJ, Cole M (1990) Proc 3th Intem Near Infrared Spectroscopy Conference. Brussels, 25-29 June, 1990, Belgium. Ed Biston and Bartiaux-Thill Jouany JP (1982) Sci Aliments 2, 131-134

Tableau I. Prévision de la teneur en acides gras volatils dans le jus de rumen (C2, C3, C4) par spectrométrie par transmission dans le proche infrarouge.

\begin{tabular}{|c|c|c|c|c|c|c|}
\hline Modèle mathématique & \multicolumn{3}{|c|}{ Longueurs d'onde sélectionnées ( $\mathrm{nm}$ ) } & \multirow{2}{*}{$\begin{array}{c}A G V \\
C 2 \\
C 3 \\
C 4\end{array}$} & \multirow{2}{*}{$\begin{array}{c}S E C^{a} \\
2,14 \\
0,63 \\
0,33\end{array}$} & \multirow{2}{*}{$\begin{array}{c}R^{2} \\
0,98 \\
0,99 \\
0,98\end{array}$} \\
\hline $\operatorname{MPLS}(2,7,6,1)$ & & & & & & \\
\hline Stepwise $(2,7,6,1)$ & $\begin{array}{l}2258 \\
2258 \\
2258\end{array}$ & $\begin{array}{l}2288 \\
2288\end{array}$ & 2378 & $\begin{array}{l}\mathrm{C} 2 \\
\mathrm{C} 2 \\
\mathrm{C} 2\end{array}$ & $\begin{array}{l}5,76 \\
4,81 \\
4,27\end{array}$ & $\begin{array}{l}0,87 \\
0,91 \\
0,93\end{array}$ \\
\hline Stepwise $(2,7,6,1)$ & $\begin{array}{l}2298 \\
2298 \\
2298\end{array}$ & $\begin{array}{l}2278 \\
2278\end{array}$ & 2328 & $\begin{array}{l}\text { C3 } \\
\text { C3 } \\
\text { C3 }\end{array}$ & $\begin{array}{l}1,51 \\
1,42 \\
1,33\end{array}$ & $\begin{array}{l}0,94 \\
0,95 \\
0,95\end{array}$ \\
\hline Stepwise $(2,7,6,1)$ & $\begin{array}{l}1668 \\
1668 \\
1668\end{array}$ & $\begin{array}{l}2238 \\
2238\end{array}$ & 2188 & $\begin{array}{l}\mathrm{C} 4 \\
\mathrm{C} 4 \\
\mathrm{C} 4\end{array}$ & $\begin{array}{l}1,41 \\
1,13 \\
0,94\end{array}$ & $\begin{array}{l}0,70 \\
0,81 \\
0,87\end{array}$ \\
\hline
\end{tabular}

- SEC : Écart type résiduel de calibration. 